\title{
Características clínicas y epidemiológicas de niños con tumores del sistema nervioso central en Medellín, Colombia
}

\author{
Juan P. Orozco-Forero ${ }^{1}$, Lina M. Martínez-Sánchez ${ }^{2 *}$, Ana P. Pamplona-Sierra², \\ Ma. de los Ángeles Rodríguez-Gázquez ${ }^{3}$, Ana C. Toro-Moreno ${ }^{4}$, Luis F. Álvarez-Hernández ${ }^{4}$, \\ Laura I. Jaramillo-Jaramillo ${ }^{4}$, Daniel Gallego-González ${ }^{4}$, Laura Serna-Vélez ${ }^{4}$ y José A. Betancur-Vergara ${ }^{5}$ \\ ${ }^{1}$ Departamento de Pediatría, Clínica Universitaria Bolivariana; ${ }^{2}$ Facultad de Medicina, Universidad Pontificia Bolivariana; ${ }^{3}$ Facultad de Enfermería, \\ Universidad de Antioquia; ${ }^{4}$ Facultad de Medicina, Escuela de Ciencias de la Salud, Facultad de Medicina, Universidad Pontificia Bolivariana; \\ ${ }^{5}$ Coordinación de Pediatría, Clínica Las Américas. Medellín, Colombia
}

\section{Resumen}

Introducción: Describir las características clínicas y epidemiológicas de los niños con diagnóstico de tumores del sistema nervioso central (SNC) atendidos en una institución de tercer nivel de complejidad de Medellín, Colombia, entre los años 2010 y 2015. Métodos: Estudio descriptivo retrospectivo, en el que se incluyó la información consignada en la historia clínica de los pacientes de 0 a 14 años con diagnóstico clínico y/o imagenológico de tumores del SNC. Resultados: Se incluyeron 85 niños con tumores del SNC, la edad promedio de diagnóstico fue de 5.7 años. Los principales hallazgos clínicos fueron cefalea (41.2\%) y las náuseas y/o vómitos (29.4\%). El $74.1 \%$ de los tumores eran de origen primario y un $8.2 \%$ secundarios a metástasis, la principal localización fue la fosa posterior con un 22.4\%. Conclusión: A pesar de que los resultados de este estudio coinciden con lo descrito en la literatura, se pudo evidenciar que hacen falta estudios epidemiológicos con una muestra mayor, que posibilite evaluar otras variables que permitan caracterizar las neoplasias de sistema nervioso en población pediátrica.

Palabras clave: Neoplasias encefálicas. Sistema nervioso central. Pediatría. Metástasis de la neoplasia. Perfil de salud.

\section{Clinical and epidemiological profile of the child population diagnosed with central nervous system cancer in Medellin, Colombia}

\begin{abstract}
Introduction: To determine the clinical and epidemiological profile of the pediatric population diagnosed with central nervous system (CNS) tumors treated in a third-level care institution in Medellín, Colombia, between 2010 and 2015. Methods: Observational, descriptive and retrospective study, which includes the information from clinical records of pediatric patients between 0 and 14 years of age with clinical diagnosis and/or imaging of CNS tumors. The analysis was carried out with the SPSS ${ }^{\circledR}$ program. Results: 85 children with CNS tumors were included; the average age of diagnosis was 5.7 years. The main clinical findings were headach $41.2 \%$, and nausea and/or vomiting $29.4 \% .74 .1 \%$ of the tumors were of primary origin and only $8.2 \%$ was secondary to metastasis; the main location was posterior fossa with $22.4 \%$. Conclusion: Despite the fact that the results of this study coincide with what has been described in the literature, it was possible to demonstrate that epidemiological studies with a larger sample are needed, which would make possible to evaluate other variables that allow the characterization of neoplasms of the nervous system in the pediatric population.
\end{abstract}

Key words: Brain neoplasms. Central nervous system. Pediatrics. Neoplasm metastasis. Health profile. 


\section{Introducción}

Los tumores del sistema nervioso central (SNC) incluyen tumores malignos y no malignos del cerebro y médula espinal, el primer grupo corresponde a la segunda causa de malignidad en niños, luego de las malignidades hematológicas, y son la causa más común de tumores de órgano sólido en pediatría; estos incluyen tumores encefálicos, de las meninges y de la médula espinal, de los cuales del 80 al 90\% corresponden a tumores cerebrales ${ }^{1-5}$. Existen dos sistemas de clasificación para categorizar los tumores del SNC en niños: la Clasificación de la Organización Mundial de la Salud según histología y parámetros moleculares y la Clasificación Internacional de Cáncer Pediátrico según el sitio del tumor primario y morfología 6 .

A diferencia de los adultos, en niños predominan los tumores primarios del SNC, y aproximadamente un tercio están localizados en fosa posterior. En EE.UU., entre los años 2008 y 2012 los principales grupos histológicos de tumores del SNC en niños fueron los gliomas, seguidos de los tumores embrionarios?.

Existen diferencias raciales y geográficas en la distribución de las neoplasias del SNC, además de diferentes factores de riesgo que pueden tener relación con la aparición de estos tumores en la infancia ${ }^{8}$. Se ha reportado que el $4 \%$ de los tumores se asocian a síndromes hereditarios o de etiología multifactorial, siendo los más comunes: neurofibromatosis tipo I y II, esclerosis tuberosa, enfermedad de von Hippel-Lindau, síndrome de poliposis familiar y síndrome de células basales nevoides ${ }^{9}$. Según la Sociedad Americana del Cáncer, «el factor de riesgo mejor conocido para el desarrollo de tumores encefálicos es la radiación, que proviene con mayor frecuencia de algún tipo de radioterapia para tratar alguna otra afección»2,10.

Los tumores del SNC pueden ocasionar signos y síntomas según el grado de invasión local, la compresión de estructuras adyacentes y el aumento de la presión intracraneal ${ }^{11-13}$.

Existen pocos datos en Antioquia sobre la epidemiología local y características clínicas de estos tumores. Por tal motivo, el objetivo planteado fue determinar el perfil clínico y epidemiológico de los niños con diagnóstico de tumores del SNC, atendidos en una institución de tercer nivel de atención de Medellín, Colombia, entre los años 2010 y 2015.

\section{Material y métodos}

Se realizó un estudio descriptivo retrospectivo, en el que se incluyó todos los pacientes de 0 y 14 años con diagnóstico clínico y/o imagenológico de tumores del SNC, que fueron atendidos en una institución de tercer nivel de complejidad en Medellín (Colombia) entre enero de 2010 y diciembre de 2015. Los investigadores utilizaron un formulario para el registro de la información obtenida de la historia clínica del paciente, que contenía datos demográficos y clínicos.

El análisis estadístico se realizó con el programa SPSS $^{\circledR}$ v.24. Se calcularon las medidas descriptivas de todas las variables: para la edad se estimó el promedio, la desviación estándar y el valor mínimo y máximo, y al resto de variables se les estimaron las frecuencias absolutas y relativas (estas últimas fueron el cociente de los casos con la característica de interés entre el número total de pacientes multiplicado por 100).

La investigación fue aprobada por el Comité de ética de las instituciones involucradas y se clasificó como una investigación sin riesgo según la Resolución 008430 de 1993 del Ministerio de Salud de Colombia.

\section{Resultados}

Se incluyeron en total 85 niños con tumores del SNC, con una edad promedio al momento del diagnóstico de $5.7 \pm 3.3$ años y una edad al momento del estudio de $8.6 \pm 3.7$ (mínimo $=1$, máximo $=14$ ). Respecto a las principales características sociodemográficas de la población, el sexo femenino representó el 55.3\%, la procedencia urbana el $57.7 \%$ y pertenecían al Régimen contributivo de Seguridad Social en Salud en Colombia un $74.1 \%$. Los antecedentes personales asociados a tumores del SNC se encontraron reportados en el $34.1 \%$ de las historias clínicas, que correspondían a un episodio convulsivo, trastorno endocrino y trauma encefalocraneano en un $20,8.2$ y $3.5 \%$ respectivamente; solo un $2.4 \%$ presentó antecedente de sarcoma.

Respecto a la presencia de signos clínicos craneofaciales, tan solo el $16.6 \%$ tenía registrada esta información. Los síntomas y signos más frecuentes en la población de estudio fueron cefalea $(41.2 \%)$, náuseas y/o vómitos (29.4\%) y síntomas motores (28.2\%).

Los métodos diagnósticos y paraclínicos que se usaron con mayor frecuencia fueron la resonancia magnética nuclear (RMN) y el hemoleucograma, con un 67.1 y un $63.5 \%$ respectivamente. Con relación a los paraclínicos se observaron resultados anormales con mayor frecuencia en el hemoleucograma, ionograma y Proteína $C$ reactiva (PCR), con un 25.9, 15.3 y $9.4 \%$ respectivamente. Respecto a las características del tumor, se observó que el $74.1 \%$ eran de origen primario y solo el $8.2 \%$ eran secundarios a metástasis; cabe resaltar que 
la principal localización fue la fosa posterior y la principal variante histológica fue el meduloblastoma, ambos con un $22.4 \%$. Se observó que al menos el $55.3 \%$ (14/85) de los tumores eran malignos, mientras que el $16.5 \%(47 / 85)$ eran benignos, el resto sin información. Según los reportes del estadio clínico, el 18.8\% (16/85) se encontraba en una etapa inicial y el $14.1 \%(12 / 85)$ en una etapa avanzada. Respecto al tipo de tratamiento empleado, la resección quirúrgica fue el más frecuente con un $87.1 \%$, seguido de la quimioterapia y la radioterapia con un 47.1 y un $21.2 \%$ respectivamente.

La principal complicación que se observó secundaria al tratamiento empleado fue la infección (no meningitis), seguida de alguna alteración neurológica, con un 15.3 y un $14.1 \%$ respectivamente. Considerando el alto porcentaje de historias clínicas sin información sobre el estado de los pacientes, la supervivencia tras seis meses del diagnóstico fue al menos del $68.2 \%$ (58/85), bajando al menos al $60 \%(51 / 85)$ a los 12 meses.

\section{Discusión}

En el estudio realizado por Azad, et al. ${ }^{14}$ en 39 niños con tumores del SNC, reportaron que el $61.5 \%$ eran de sexo masculino, con un promedio de edad de 10 años, diferente a lo encontrado en los resultados previamente presentados, donde predominó el sexo femenino, con un $55.3 \%$, y se obtuvo un promedio de edad de 5.7 años. En el estudio realizado en Cartagena, Colombia, por Ramos-Clason, et al. ${ }^{5}$, reportaron un $53.4 \%$ de sexo masculino con un promedio de edad de 8 años en 58 niños con tumores en SNC. Por otra parte, un estudio iraní con 198 niños encontró que el $63.1 \%$ (125) correspondió al sexo masculino, resultado concordante con lo expuesto por Azad, et al. ${ }^{15}$. En cuanto a las manifestaciones clínicas, la cefalea y la hemiparesia fueron las reportadas con mayor frecuencia por Azad, et al. ${ }^{14}$, con un 27 y un $24.3 \%$ respectivamente, cifras comparables con los datos observados por nuestro equipo, donde los principales síntomas fueron la cefalea y náuseas y/o vómito con un 41.2 y un $29.4 \%$ respectivamente; contrario al estudio realizado por Suresh, et al. ${ }^{16}$ en el sur de la India, donde el principal síntoma fue el vómito con un $62.5 \%$, seguido de la cefalea en un $37.5 \%$. Concordante con lo encontrado por Suresh, et al..$^{16}$, en el estudio de Faranoush, et al. ${ }^{15}$ el vómito $(n=106,53.5 \%)$ y la cefalea ( $n=102,51.5 \%)$ fueron los principales hallazgos clínicos. Las indicaciones para realizar una neuroimagen se basan en el índice de sospecha respecto a síntomas y signos evidenciados en un paciente, es por esto que en todo niño que presente cefalea persistente (continua o recurrente, mayor a 4 semanas) se debe sospechar un tumor del SNC, y se requiere de una neuroimagen para descartarlo ${ }^{17,18}$. Los resultados expuestos en este manuscrito evidencian que se realizó RMN en un $67 \%$ de los niños y tomografía computarizada en un $57 \%$. La biopsia solo se obtuvo en un $37 \%$ de los casos. En relación con la localización de los tumores cerebrales, se encontró una frecuencia de tumores de fosa posterior en un $22.4 \%$, similar a lo reportado por Ostrom, et al. ${ }^{7}$, cuyas cinco localizaciones más frecuentes fueron: cerebelo $18.5 \%$, otras regiones cerebrales $15.2 \%$, tallo cerebral $12.4 \%$, seguido de tumores pituitarios, del ducto craneofaríngeo $8.1 \%$ y nervios craneales $7.5 \%$. Respecto a las variantes histológicas, Azad, et al. ${ }^{14}$ reportaron que el más frecuente fue el ependimoma, con un $19.4 \%$, seguido por el astrocitoma pilocítico y el meduloblastoma con $16.7 \%$ cada uno, mientras que en nuestros hallazgos el más frecuente fue el meduloblastoma con $22.4 \%$, seguido del astrocitoma pilocítico y tumor neuroectodérmico con 16.5 y $8.2 \%$, respectivamente; de igual forma, Shirazi, et al. ${ }^{19}$ encontraron el meduloblastoma ( $n=11,20.37 \%$ ) como principal neoplasia. Por su parte, Zhou, et al. ${ }^{20}$ en su estudio realizado en China con 1.485 casos, hallaron como tumores del SNC más frecuentes los astrocitomas (31\%), craniofaringiomas $(18 \%)$, meduloblastoma (15\%), tumores de células germinales $(8 \%)$ y tumores ependimales (6\%). Los resultados arrojados en el estudio de Faranoush, et al. ${ }^{15}$ muestran el glioma de alto grado y el meduloblastoma como las principales neoplasias encontradas, con un 59.99 y un $48.51 \%$ de los tumores supratentoriales e infratentoriales respectivamente. El equipo de investigación encontró que el principal tratamiento fue la cirugía, realizada en el $87.1 \%$, dato similar a lo reportado por Azad, et al. ${ }^{14}$, un $80 \%$, de los cuales el $52.5 \%$ requirió cirugías adicionales, mientras que en nuestra población solo $14.1 \%$ fueron reintervenidos. Igualmente, en el estudio realizado por Suresh, et al. ${ }^{16}$ la cirugía fue descrita en el $69 \%$ de los casos. Respecto a otro tipo de tratamiento, Suresh, et al. ${ }^{16}$ reportaron la radioterapia y quimioterapia en un 53.8 y un $50 \%$ respectivamente; datos disimiles a los reportados aquí, donde la quimioterapia y la radioterapia se reportaron en el 47.1 y el $21.2 \%$, respectivamente. La radioterapia y la quimioterapia son tratamientos adyuvantes y se basan en la histología del tumor ${ }^{21}$. La tasa de supervivencia depende de cada tumor, en EE.UU. la supervivencia a 5 y 10 años para niños con tumores 
del SNC fue del 73 y el $70 \%{ }^{7}$. Los niños que sobreviven a tumores del SNC generalmente permanecen con secuelas neurológicas, cognitivas, psicológicas y endocrinológicas secundarias al tumor por sí mismo y al tratamiento usado. El riesgo de estas secuelas aumenta en pacientes de menor edad (menores de 3 años) en el momento del diagnóstico y tratamiento, con hidrocefalia y/o tratamiento previo con radioterapia en cráneo ${ }^{22}$.

\section{Conclusión}

Con este estudio se puede evidenciar el perfil clínico y epidemiológico de 85 niños con tumores del SNC atendidos en una institución de Medellín, donde los principales síntomas fueron cefalea y náuseas y/o vómito. La principal variante histológica para esta población fue el meduloblastoma, a diferencia de otros estudios realizados en otros países desarrollados y en vías de desarrollo. La tasa de supervivencia de estos pacientes a un año fue del $60 \%$, menor a lo reportado previamente. Sin embargo, hacen falta más estudios epidemiológicos locales donde se incluyan un mayor número de pacientes, en los que se permita realizar un seguimiento a largo plazo para evaluar la presencia de secuelas transitorias y permanentes y la supervivencia tras más años del diagnóstico y tratamiento ofrecido.

\section{Financiamiento}

El financiamiento del proyecto estuvo a cargo de la Universidad Pontificia Bolivariana.

\section{Conflicto de intereses}

Los autores declaran no tener ningún conflicto de intereses

\section{Responsabilidades éticas}

Protección de personas y animales Los autores declaran que para esta investigación no se han realizado experimentos en seres humanos ni en animales.

Confidencialidad de los datos Los autores declaran que han seguido los protocolos de su centro de trabajo sobre la publicación de datos de pacientes.

Derecho a la privacidad y consentimiento informado Los autores declaran que en este artículo no aparecen datos de pacientes.

\section{Bibliografía}

1. Linabery AM, Ross JA. Trends in childhood cancer incidence in the U.S. (1992-2004). Cancer. 2008;112(2):416-32.

2. Morales M, Sánchez-Vergara AL, Rubio-López N, Llopis-González A. Exposición a factores ambientales y riesgo de tumores en el sistema nervioso central en niños: revisión sistemática y meta-análisis. Rev Salud Ambient. 2014;14(2):74-86.

3. Arias Andrade MV, Maya Hijuelos LC (dir), Godoy Cordobés JA (dir), Vásquez Hoyos $\mathrm{P}$ (dir). Caracterización de los pacientes atendidos en la Unidad de Cuidado Intensivo pediátrica del Instituto Nacional de Cancerología, Bogotá, Colombia [Tesis para título de especialista en Internet]. [Bogotá, Colombia]: Universidad Nacional de Colombia, Facultad de Medicina, Departamento de Pediatría; 2014. Disponible en: http:// bdigital.unal.edu.co/12443/1/5599667.2014.pdf

4. Pardo C, Cendales R. Incidencia, mortalidad y prevalencia de cáncer en Colombia, 2007-2011. Primera edición. Bogotá D.C.: Instituto Nacional de Cancerología; 2015.

5. Ramos-Clason E, Tuñón-Pitalua M, Rivas-Muñoz F, Veloza-Cabrera L. Tumores primarios del sistema nervioso central en Cartagena, 20012006. Rev Salud Pública. 2010;12(2):257-67.

6. Louis DN, Perry A, Reifenberger G, von Deimling A, Figarella-Branger D, Cavenee WK, et al. The 2016 World Health Organization Classification of Tumors of the Central Nervous System: a summary. Acta Neuropathol. 2016;131(6):803-20.

7. Ostrom QT, Gittleman H, Fulop J, Liu M, Blanda R, Kromer C, et al. CBTRUS Statistical Report: Primary brain and central nervous system tumors diagnosed in the United States in 2008-2012. Neuro Oncol. 2015;17(Suppl 4):iv1-iv62.

8. Villarejo F, Martínez J. Tumores cerebrales en niños. Pediatr Integral. 2012;XVI(6):475-86.

9. Martínez González MJ, García Ribes A, Garaizar Axpe C. Tumores cerebrales infantiles: diagnóstico y semiología neurológica [Internet]. Madrid: Asociación Española de Pediatría; 2008 [consultado: 16 de enero de 2018]. Disponible en: https://www.aeped.es/sites/default/files/documentos/27-tumores.pdf

10. Taylor AJ, Little MP, Winter DL, Sugden E, Ellison DW, Stiller CA, et al. Population-based risks of CNS tumors in survivors of childhood cancer: the British Childhood Cancer Survivor Study. J Clin Oncol. 2010;28(36):5287-93.

11. Wilne SH, Dineen RA, Dommett RM, Chu TP, Walker DA. Identifying brain tumours in children and young adults. BMJ. 2013;347:£5844.

12. Fischman $M$, Ismael J, Pesce $P$, Rufach $D$. Tumores pediátricos del sistema nervioso central [Internet]. Argentina: Instituto nacional de Cáncer; 2015 [consultado: 16 de enero de 2018]. Disponible en: http://www.msal.gob.ar/ images/stories/bes/graficos/0000000811cnt-67-tumores-del-SNC.pdf

13. Wilne S, Collier J, Kennedy C, Koller K, Grundy R, Walker D. Presentation of childhood CNS tumours: a systematic review and meta-analysis. Lancet Oncol. 2007;8(8):685-95.

14. Azad TD, Shrestha RK, Vaca S, Niyaf A, Pradhananga A, Sedain G, et al. Pediatric central nervous system tumors in Nepal: Retrospective analysis and literature review of low- and middle-income countries. World Neurosurg. 2015;84(6):1832-7.

15. Faranoush M, Torabi-Nami M, Mehrvar A, HedayatiAsl AA, Tashvighi M, Parsa RR, et al. Classifying pediatric central nervous system tumors through near optimal feature selection and mutual information: A single center cohort. Middle East J Cancer. 2013;4(4):153-62.

16. Suresh SG, Srinivasan A, Scott JX, Rao SM, Chidambaram B, Chandrasekar S. Profile and outcome of pediatric brain tumors - Experience from a tertiary care pediatric oncology unit in South India. J Pediatr Neurosci. 2017;12(3):237-44.

17. Matthews PM, Wylezinska M, Cadoux-Hudson T. Novel approaches to imaging brain tumors. Hematol Oncol Clin North Am. 2001; 15(4):609-30.

18. Wilne S, Koller K, Collier J, Kennedy C, Grundy R, Walker D. The diagnosis of brain tumours in children: a guideline to assist healthcare professionals in the assessment of children who may have a brain tumour. Arch Dis Child. 2010;95(7):534-9.

19. Shirazi N, Gupta M, Bhat NK, Kalra BP, Kumar R, Saini M. Profile of primary pediatric brain and spinal cord tumors from North India. Indian J Med Paediatr Oncol. 2017;38:10-4.

20. Zhou D, Zhang Y, Liu H, Luo S, Luo L, Dai K. Epidemiology of nervous system tumors in children: a survey of 1,485 cases in Beijing Tiantan Hospital from 2001 to 2005. Pediatr Neurosurg. 2008;44(2):97-103.

21. Blaney SM, Hass-Kogan D, Poussaint TY, Santi M, Gilbertson R, Parsons DW, et al. Gliomas, ependymomas, and other nonembryonal tumors of the central nervous system. En: Pizzo P, Poplack D (editores). Principles and practice of pediatric oncology, 6 th ed. Philadelphia: Wolters Kluwer Health/Lippincott Williams \& Wilkins; 2011. p. 717.

22. Fouladi M, Gilger E, Kocak M, Wallace D, Buchanan G, Reeves C, et al. Intellectual and functional outcome of children 3 years old or younger who have CNS malignancies. J Clin Oncol. 2005;23(28):7152-60. 